\title{
A monotonicity formula for stationary biharmonic maps
}

\section{Journal Article}

Author(s):

Angelsberg, Gilles

Publication date:

2006-02

Permanent link:

https://doi.org/10.3929/ethz-b-000412024

Rights / license:

In Copyright - Non-Commercial Use Permitted

Originally published in:

Mathematische Zeitschrift 252(2), https://doi.org/10.1007/s00209-005-0848-z 


\title{
A monotonicity formula for stationary biharmonic maps $^{\star}$
}

\author{
Gilles Angelsberg \\ Departement für Mathematik, ETH Zentrum, Rämistrasse 101, CH-8092 Zürich, \\ Switzerland (e-mail: gilles@math.ethz.ch)
}

Received: 29 October 2004; in final form: 7 February 2005 /

Published online: 16 August 2005 - (C) Springer-Verlag 2005

\begin{abstract}
We give a rigorous proof of the monotonicity formula of S.-Y.A. Chang, L. Wang and P. Yang [3] for (extrinsically) stationary biharmonic maps of class $W^{2,2}$.
\end{abstract}

\section{Introduction}

For several decades regularity properties of weakly harmonic maps have been intensely studied. For a manifold $M$ of dimension $m \leq 2$, C.B. Morrey [9] showed in 1948 that every minimizing map $u \in W^{1,2}(M, N)$ belongs to $C^{\infty}(M, N)$. The regularity problem for general critical points of the harmonic energy functional had remained open for a long time. In 1981, still for the case $m \leq 2$, M. Grüter [5] proved smoothness of conformal weakly harmonic maps. R. Schoen [12] introduced the notion of stationary harmonic maps and extended Grüter's result to this class. Finally, F. Hélein [6] showed that every weakly harmonic map in the case $m \leq 2$ is smooth. For $m \geq 3$, more complex phenomena show up. R. Schoen and $\mathrm{K}$. Uhlenbeck [13] showed that if $u \in W^{1,2}(M, N)$ is energy minimizing, then $\mathrm{u}$ is smooth except on a closed subset of finite $H^{m-3}$-measure. This result is optimal since the radial projection from $B^{m}$ into $S^{m-1}$ is a minimizing map for $m \geq 3$, as shown by H. Brézis, J.-M. Coron and E. Lieb [2] for $m=3$ and F.H. Lin [8] for $m \geq 3$. On the other hand, T. Rivière [11] proved existence of everywhere discontinuous weakly harmonic maps. For the intermediate class of stationary harmonic maps, L.C. Evans [4] showed partial regularity for maps into the sphere and F. Bethuel [1] generalized this result for arbitrary target manifolds. Their proofs rely on a monotonicity formula for stationary harmonic maps adapted from P. Price [10].

^ This work was partially supported by SNF 200021-101930/1. 
Similar questions for (extrinsically) weakly biharmonic maps were studied by S.-Y.A. Chang, L. Wang and P. Yang in [3]. They showed smoothness for weakly biharmonic maps into the sphere and $m \leq 4$, and asserted partial regularity for stationary biharmonic maps into the sphere and $m \geq 5$. C.Y. Wang generalized these results for arbitrary target manifolds in [14] and [15]. Once again, a monotonicity formula derived from the stationarity assumption is crucial in the proof of partial regularity for $m \geq 5$. However, the derivation in [3, Proposition 3.2] is only given for sufficiently regular maps.

Here, we give a rigorous proof of this monotonicity formula in the case of stationary biharmonic maps of class $W^{2,2}\left(B_{r}, N\right)$.

Conceivably, this monotonicity formula may allow to study the singular behaviour of stationary biharmonic maps and especially minimizing biharmonic maps as suggested by M-C. Hong and C.Y. Wang in [7].

\section{Stationarity Assumption}

We recall some definitions and fix the notations used henceforth: Let $B_{r}$ be the open ball of radius $r>0$ in $\mathbb{R}^{m}$ centered at the origin and let $N$ be a smooth Riemannian manifold isometrically embedded in $\mathbb{R}^{n}$. Recall that $W^{2,2}\left(B_{r}, N\right)$ is defined by

$$
W^{2,2}\left(B_{r}, N\right):=\left\{u \in W^{2,2}\left(B_{r}, \mathbb{R}^{n}\right): u(x) \in N \text { for a.e. } x \in B_{r}\right\} .
$$

We consider on $W^{2,2}\left(B_{r}, N\right)$ the Hessian energy functional

$$
E(u):=\int_{B_{r}}|\Delta u|^{2} d x .
$$

Definition 1. A map u in $W^{2,2}\left(B_{r}, N\right)$ is called (extrinsically) weakly biharmonic if it is a critical point of the Hessian energy $E(\cdot)$ with respect to compactly supported variations on the target manifold, i.e. if

$$
\left.\frac{d}{d t}\right|_{t=0} E(\pi \circ(u+t \xi))=0 \text { for all } \xi \in \mathcal{C}_{0}^{\infty}\left(B_{r}, \mathbb{R}^{n}\right),
$$

where $\pi$ denotes the nearest point projection onto $N$.

Definition 2. A weakly biharmonic map u in $W^{2,2}\left(B_{r}, N\right)$ is called (extrinsically) stationary biharmonic if, in addition, $u$ is a critical point of the Hessian energy $E(\cdot)$ with respect to compactly supported variations on the domain manifold, i.e. if

$$
\left.\frac{d}{d t}\right|_{t=0} E(u \circ(i d+t \xi))=0 \text { for all } \xi \in \mathcal{C}_{0}^{\infty}\left(B_{r}, \mathbb{R}^{m}\right),
$$

where id denotes the identity map.

In what follows we denote $u=\left(u^{1}, \ldots, u^{n}\right), u_{j}=\frac{\partial u}{\partial x_{j}}$ etc. We tacitly sum over repeated indices. We use the following notation

$$
\int_{\partial B_{r} \backslash \partial B_{\rho}} f d \sigma:=\int_{\partial B_{r}} f d \sigma-\int_{\partial B_{\rho}} f d \sigma .
$$

We replace Lemma 3.1 in [3] by the following lemma. 
Lemma 1. If $u$ is a stationary biharmonic map in $W^{2,2}\left(B_{2 r}, N\right)$, then we have:

$$
\int_{B_{2 r}}\left(4 u_{k k} u_{i j} \xi_{i}^{j}+2 u_{k k} u_{j} \xi_{i i}^{j}-|\Delta u|^{2} \nabla \cdot \xi\right) d x=0
$$

for every test function $\xi \in \mathcal{C}_{0}^{\infty}\left(B_{2 r}, \mathbb{R}^{m}\right)$.

Proof. We compute

$$
\Delta u_{t}=(\Delta u)_{t}+t\left(2\left(u_{i j}\right)_{t} \xi_{i}^{j}+\left(u_{j}\right)_{t} \xi_{i i}^{j}\right)+t^{2}\left(u_{j k}\right)_{t} \xi_{i}^{k} \xi_{i}^{j},
$$

where for any $f$ we denote $f_{t}(y):=f(y+t \xi(y))$ for $\xi \in \mathcal{C}_{0}^{\infty}\left(B_{2 r}, \mathbb{R}^{m}\right)$. The change of variables $x=y+t \xi$ for $|t|$ sufficiently small induces a $\mathcal{C}^{\infty}$ diffeomorphism of $B_{2 r}$ onto itself. The stationarity assumption (1) then implies:

$$
\begin{aligned}
0 & =\left.\frac{d}{d t}\right|_{t=0} \int_{B_{2 r}}\left|\Delta u_{t}\right|^{2}(x-t \xi)\left(1-t \nabla \cdot \xi+O\left(t^{2}\right)\right) d x \\
& =\int_{B_{2 r}}\left(4 u_{k k} u_{i j} \xi_{i}^{j}+2 u_{k k} u_{j} \xi_{i i}^{j}-|\Delta u|^{2} \nabla \cdot \xi\right) d x .
\end{aligned}
$$

\section{Monotonicity formula}

We can now rigorously derive the monotonicity formula Proposition 3.2. in [3] for almost every radius. This is sufficient to prove partial regularity for (extrinsically) stationary biharmonic maps of class $W^{2,2}$.

Theorem 1 (Monotonicity formula). For $K>0$ and $u \in W^{2,2}\left(B_{K}, N\right)$ (extrinsically) stationary biharmonic, it holds for a.e. $0<\rho<r \leq \frac{K}{2}$

$$
r^{4-m} \int_{B_{r}}|\Delta u|^{2} d x-\rho^{4-m} \int_{B_{\rho}}|\Delta u|^{2} d x=P+R,
$$

where

$$
\begin{aligned}
& P=4 \int_{B_{r} \backslash B_{\rho}}\left(\frac{\left(u_{j}+x^{i} u_{i j}\right)^{2}}{|x|^{m-2}}+\frac{(m-2)\left(x^{i} u_{i}\right)^{2}}{|x|^{m}}\right) d x \\
& R=2 \int_{\partial B_{r} \backslash \partial B_{\rho}}\left(-\frac{x^{i} u_{j} u_{i j}}{|x|^{m-3}}+2 \frac{\left(x^{i} u_{i}\right)^{2}}{|x|^{m-1}}-2 \frac{|\nabla u|^{2}}{|x|^{m-3}}\right) d \sigma .
\end{aligned}
$$

Proof. Choose in Lemma 1 the test functions $\xi(x):=\psi^{\epsilon}\left(\frac{|x|}{\tau}\right) x$, where $\psi=\psi^{\epsilon}$ : $\mathbb{R}_{+} \rightarrow[0,1]$ is smooth with compact support on $[0,1]$ and $\psi^{\epsilon} \equiv 1$ on $[0,1-\epsilon]$. Then, it follows with $\xi_{i}^{j}=\psi_{i} x^{j}+\psi \delta_{i j}$ and $\xi_{i i}^{j}=\psi_{i i} x^{j}+2 \psi_{j}$

$$
\begin{gathered}
0=\int_{\mathbb{R}^{m}}\left((4-m)|\Delta u|^{2} \psi-|\Delta u|^{2} \psi_{i} x^{i}+4 u_{k k} u_{i j} \psi_{i} x^{j}\right. \\
\left.+4 u_{k k} u_{j} \psi_{j}+2 u_{k k} u_{j} \psi_{i i} x^{j}\right) d x
\end{gathered}
$$

We have $\psi_{i}\left(\frac{|x|}{\tau}\right)=\frac{1}{\tau} \psi^{\prime}\left(\frac{|x|}{\tau}\right) \frac{x^{i}}{|x|}$ and $\psi_{i i}\left(\frac{|x|}{\tau}\right)=\frac{1}{\tau^{2}} \psi^{\prime \prime}\left(\frac{|x|}{\tau}\right)+\frac{(m-1)}{\tau} \psi^{\prime}\left(\frac{|x|}{\tau}\right) \frac{1}{|x|}$. 
Thus,

$$
\begin{aligned}
0= & (4-m) \int_{\mathbb{R}^{m}}|\Delta u|^{2} \psi d x-\frac{1}{\tau} \int_{\mathbb{R}^{m}}|\Delta u|^{2} \psi^{\prime}|x| d x+\frac{4}{\tau} \int_{\mathbb{R}^{m}} u_{k k} u_{i j} \psi^{\prime} \frac{x^{i} x^{j}}{|x|} d x \\
& +\frac{2(m+1)}{\tau} \int_{\mathbb{R}^{m}} u_{k k} u_{j} \psi^{\prime} \frac{x^{j}}{|x|} d x+\frac{2}{\tau^{2}} \int_{\mathbb{R}^{m}} u_{k k} u_{j} \psi^{\prime \prime} x^{j} d x .
\end{aligned}
$$

This implies for $I^{\epsilon}(\tau):=\tau^{4-m} \int_{\mathbb{R}^{m}}|\Delta u|^{2} \psi^{\epsilon}\left(\frac{|x|}{\tau}\right) d x$

$$
\begin{aligned}
\tau^{m-3} \frac{d}{d \tau} I^{\epsilon}(\tau)= & (4-m) \int_{\mathbb{R}^{m}}|\Delta u|^{2} \psi d x-\frac{1}{\tau} \int_{\mathbb{R}^{m}}|\Delta u|^{2} \psi^{\prime}|x| d x \\
= & -\frac{4}{\tau} \int_{\mathbb{R}^{m}} u_{k k} u_{i j} \psi^{\prime} \frac{x^{i} x^{j}}{|x|} d x-\frac{2(m+1)}{\tau} \int_{\mathbb{R}^{m}} u_{k k} u_{j} \psi^{\prime} \frac{x^{j}}{|x|} d x \\
& -\frac{2}{\tau^{2}} \int_{\mathbb{R}^{m}} u_{k k} u_{j} \psi^{\prime \prime} x^{j} d x .
\end{aligned}
$$

Furthermore, we have:

$$
\begin{aligned}
\int_{\rho}^{r} \psi^{\prime \prime}\left(\frac{|x|}{\tau}\right) \tau^{1-m} d \tau= & -\int_{\rho}^{r} \frac{d}{d \tau}\left(\psi^{\prime}\left(\frac{|x|}{\tau}\right)\right) \tau^{3-m} \frac{1}{|x|} d \tau \\
= & -\psi^{\prime}\left(\frac{|x|}{r}\right) \frac{r^{3-m}}{|x|}+\psi^{\prime}\left(\frac{|x|}{\rho}\right) \frac{\rho^{3-m}}{|x|} \\
& +\frac{3-m}{|x|} \int_{\rho}^{r} \psi^{\prime}\left(\frac{|x|}{\tau}\right) \tau^{2-m} d \tau
\end{aligned}
$$

Thus, applying Fubini's theorem twice gives:

$$
\begin{aligned}
& \int_{\rho}^{r} \tau^{1-m} \int_{\mathbb{R}^{m}} u_{k k} u_{j} \psi^{\prime \prime}\left(\frac{|x|}{\tau}\right) x^{j} d x d \tau \\
& =-\int_{\mathbb{R}^{m}} u_{k k} u_{j} x^{j} \psi^{\prime}\left(\frac{|x|}{r}\right) \frac{r^{3-m}}{|x|} d x+\int_{\mathbb{R}^{m}} u_{k k} u_{j} x^{j} \psi^{\prime}\left(\frac{|x|}{\rho}\right) \frac{\rho^{3-m}}{|x|} d x \\
& \quad+(3-m) \int_{\rho}^{r} \tau^{2-m} \int_{\mathbb{R}^{m}} u_{k k} u_{j} x^{j} \psi^{\prime}\left(\frac{|x|}{\tau}\right) \frac{1}{|x|} d x d \tau .
\end{aligned}
$$

Multiplying both sides of (2) with $\tau^{3-m}$, integrating over $\tau$ from $\rho$ to $r$ and inserting (3) yield

$$
\begin{aligned}
I^{\epsilon}(r)-I^{\epsilon}(\rho)= & -4 \int_{\rho}^{r} \tau^{2-m} \int_{\mathbb{R}^{m}} u_{k k} u_{i j} \psi^{\prime}\left(\frac{|x|}{\tau}\right) \frac{x^{i} x^{j}}{|x|} d x d \tau \\
& -8 \int_{\rho}^{r} \tau^{2-m} \int_{\mathbb{R}^{m}} u_{k k} u_{j} \psi^{\prime}\left(\frac{|x|}{\tau}\right) \frac{x^{j}}{|x|} d x d \tau \\
& +2 \int_{\mathbb{R}^{m}} u_{k k} u_{j} x^{j} \psi^{\prime}\left(\frac{|x|}{r}\right) r^{3-m} \frac{1}{|x|} d x \\
& -2 \int_{\mathbb{R}^{m}} u_{k k} u_{j} x^{j} \psi^{\prime}\left(\frac{|x|}{\rho}\right) \rho^{3-m} \frac{1}{|x|} d x .
\end{aligned}
$$

For all Lebesgue points $\rho$ and $r$ of the function $\left.\left.g(s):=\int_{\partial B_{s}} \frac{u_{k k} u_{j} x^{j}}{|x|^{m-3}} d \sigma \in L_{\text {loc }}^{1}(] 0, K\right]\right)$, as $\epsilon \rightarrow 0$, by applying Lemma 2 in the Appendix to the first two terms and the Lebesgue differentiation theorem to the last two terms, we obtain 


$$
\begin{aligned}
r^{4-} & \int_{B_{r}}|\Delta u|^{2} d x-\rho^{4-m} \int_{B_{\rho}}|\Delta u|^{2} d x \\
& =\int_{B_{r} \backslash B_{\rho}}\left(4 \frac{u_{k k} u_{i j} x^{i} x^{j}}{|x|^{m-2}}+8 \frac{u_{k k} u_{j} x^{j}}{|x|^{m-2}}\right) d x-2 \int_{\partial B_{r} \backslash \partial B_{\rho}} \frac{u_{k k} u_{j} x^{j}}{|x|^{m-3}} d \sigma \\
& =2 \int_{B_{r} \backslash B_{\rho}}\left(\frac{u_{k k} u_{i j} x^{i} x^{j}}{|x|^{m-2}}-\frac{u_{k k i} u_{j} x^{i} x^{j}}{|x|^{m-2}}+\frac{u_{k k} u_{j} x^{j}}{|x|^{m-2}}\right) d x .
\end{aligned}
$$

Here and henceforth, all third derivatives are interpreted in the sense of distributions. The monotonicity formula now follows by several integrations by parts. We proceed as in [3] and compute for a.e. $\rho$ and $r$

$$
\begin{aligned}
& \int_{B_{r} \backslash B_{\rho}} \frac{u_{k k} u_{i j} x^{i} x^{j}}{|x|^{m-2}} d x=\int_{\partial B_{r} \backslash \partial B_{\rho}} \frac{u_{k} u_{i j} x^{i} x^{j} x^{k}}{|x|^{m-1}} d \sigma-\int_{B_{r} \backslash B_{\rho}} \frac{u_{k} u_{i j k} x^{i} x^{j}}{|x|^{m-2}} d x \\
& +\int_{B_{r} \backslash B_{\rho}}\left(-2 \frac{u_{k} u_{i k} x^{i}}{|x|^{m-2}}+\frac{(m-2) u_{k} u_{i j} x^{i} x^{j} x^{k}}{|x|^{m}}\right) d x \\
& =\int_{\partial B_{r} \backslash \partial B_{\rho}}\left(\frac{u_{k} u_{i j} x^{i} x^{j} x^{k}}{|x|^{m-1}}-\frac{u_{k} u_{i k} x^{i}}{|x|^{m-3}}\right) d \sigma \\
& +\int_{B_{r} \backslash B_{\rho}}\left(\frac{u_{k} u_{i k} x^{i}}{|x|^{m-2}}+\frac{u_{i k} u_{j k} x^{i} x^{j}}{|x|^{m-2}}\right) d x \\
& +(m-2) \int_{B_{r} \backslash B_{\rho}} \frac{u_{k} u_{i j} x^{i} x^{j} x^{k}}{|x|^{m}} d x, \\
& \int_{B_{r} \backslash B_{\rho}}-\frac{u_{k k i} u_{j} x^{i} x^{j}}{|x|^{m-2}} d x=-\int_{\partial B_{r} \backslash \partial B_{\rho}} \frac{u_{k i} u_{j} x^{i} x^{j} x^{k}}{|x|^{m-1}} \\
& +\int_{B_{r} \backslash B_{\rho}}\left(\frac{u_{k j} u_{j} x^{k}}{|x|^{m-2}}+\frac{u_{j} u_{k k} x^{j}}{|x|^{m-2}}\right) d x \\
& +\int_{B_{r} \backslash B_{\rho}}\left(\frac{(2-m) u_{j} u_{i k} x^{i} x^{j} x^{k}}{|x|^{m}}+\frac{u_{i k} u_{j k} x^{i} x^{j}}{|x|^{m-2}}\right) d x, \\
& \int_{B_{r} \backslash B_{\rho}} \frac{u_{k k} u_{j} x^{j}}{|x|^{m-2}} d x=\int_{\partial B_{r} \backslash \partial B_{\rho}} \frac{\left(u_{k} x^{k}\right)^{2}}{|x|^{m-1}} d \sigma-\int_{B_{r} \backslash B_{\rho}} \frac{|\nabla u|^{2}}{|x|^{m-2}} d x \\
& +\int_{B_{r} \backslash B_{\rho}}\left(\frac{(m-2)\left(u_{k} x^{k}\right)^{2}}{|x|^{m}}-\frac{u_{k} u_{j k} x^{j}}{|x|^{m-2}}\right) d x .
\end{aligned}
$$

Combining these equations leads to

$$
\begin{aligned}
r^{4-m} & \int_{B_{r}}|\Delta u|^{2} d x-\rho^{4-m} \int_{B_{\rho}}|\Delta u|^{2} d x \\
= & 2 \int_{\partial B_{r} \backslash \partial B_{\rho}}\left(\frac{\left(u_{i} x^{i}\right)^{2}}{|x|^{m-1}}-\frac{u_{j} u_{i j} x^{i}}{|x|^{m-3}}\right) d \sigma \\
& +2 \int_{B_{r} \backslash B_{\rho}}\left(\frac{u_{j} u_{i j} x^{i}}{|x|^{m-2}}+2 \frac{\left(u_{i j} x^{i}\right)^{2}}{|x|^{m-2}}+\frac{u_{i} u_{j j} x^{i}}{|x|^{m-2}}-\frac{|\nabla u|^{2}}{|x|^{m-2}}+\frac{(m-2)\left(u_{i} x^{i}\right)^{2}}{|x|^{m}}\right) d x .
\end{aligned}
$$


Moreover, it holds

$$
0=-4 \int_{\partial B_{r} \backslash \partial B_{\rho}} \frac{|\nabla u|^{2}}{|x|^{m-3}} d \sigma+8 \int_{B_{r} \backslash B_{\rho}}\left(\frac{|\nabla u|^{2}}{|x|^{m-2}}+\frac{u_{j} u_{i j} x^{i}}{|x|^{m-2}}\right) d x
$$

and

$$
\begin{aligned}
0= & 2 \int_{\partial B_{r} \backslash \partial B_{\rho}} \frac{\left(u_{i} x^{i}\right)^{2}}{|x|^{m-1}} d \sigma \\
& -2 \int_{B_{r} \backslash B_{\rho}}\left(\frac{u_{j} u_{i j} x^{i}}{|x|^{m-2}}+\frac{|\nabla u|^{2}}{|x|^{m-2}}+\frac{u_{i} u_{j j} x^{i}}{|x|^{m-2}}+\frac{(2-m)\left(u_{i} x^{i}\right)^{2}}{|x|^{m}}\right) d x .
\end{aligned}
$$

Adding the last three equations establishes the monotonicity formula.

\section{Appendix}

For $f, \phi \in L^{1}(\mathbb{R}, \mathbb{R})$, define

$$
(\phi * f)(x):=\int_{\mathbb{R}} \phi\left(\frac{y}{x}\right) f(y) d y=\int_{\mathbb{R}} \phi(z) f(x z) x d z .
$$

Then, there holds the following

Lemma 2. Suppose $f,\left(\phi_{k}\right)_{k \in \mathbb{N}} \in L^{1}(\mathbb{R}, \mathbb{R})$, with

$$
\phi_{k} \geq 0, \int_{\mathbb{R}} \phi_{k}=1 \text { and } \operatorname{supp} \phi_{k} \subset\left[1-\frac{1}{k}, 1\right], k \in \mathbb{N} \text {. }
$$

Then, as $k \rightarrow \infty$ for $0<\rho<r$

$$
\phi_{k} * f \rightarrow i d \cdot f \text { in } L^{1}([\rho, r]),
$$

where $(i d \cdot f)(x)=x f(x)$.

Proof. Observe that

$$
\|f(h \cdot)-f\|_{L^{1}(\mathbb{R}, \mathbb{R})} \rightarrow 0 \text { for } h \rightarrow 1
$$

and

$$
\left(\phi_{k} * f\right)(x)-x f(x)=x \int_{\mathbb{R}} \phi_{k}(z)(f(x z)-f(x)) d z .
$$

Thus, we have using Fubini's theorem and Hölder's inequality:

$$
\begin{aligned}
\left\|\phi_{k} * f-x f\right\|_{L^{1}([\rho, r])} & \leq \int_{\rho}^{r}|x| \int_{\mathbb{R}}\left|\phi_{k}(z)\right||f(x z)-f(x)| d z d x \\
& \leq r \int_{\mathbb{R}}\left|\phi_{k}(z)\right|\left(\int_{\rho}^{r}|f(x z)-f(x)| d x\right) d z \\
& \leq r\left\|\phi_{k}\right\|_{L^{1}} \sup _{h \in \operatorname{supp} \phi_{k}}\|f(h \cdot)-f\|_{L^{1}} .
\end{aligned}
$$

As $\operatorname{supp} \phi_{k} \subset\left[1-\frac{1}{k}, 1\right]$ and $\left\|\phi_{k}\right\|_{L^{1}} \leq 1$, assumption (4) implies that the right hand side converges to zero for $k \rightarrow \infty$. 
Acknowledgements. I would like to thank Michael Struwe for his many helpful discussions.

\section{References}

1. Bethuel, F.: On the singular set of stationary harmonic maps. Manuscripta Math. 78, 417-443 (1993)

2. Brézis, H., Coron, J.-M., Lieb, E.: Harmonic maps with defects. Comm. Math. Phys. 107, 649-705 (1986)

3. Chang, S.-Y.A., Wang, L., Yang, P.: A regularity theory for biharmonic maps. Comm. Pure Appl. Math. 52, 1113-1137 (1999)

4. Evans, L.C.: Partial regularity for stationary harmonic maps into the sphere. Arch. Rational Mech. Anal. 116, 101-113 (1991)

5. Grüter, M.: Regularity of weak H-surfaces. J. reine angew. Math. 329, 1-15 (1981)

6. Hélein, F.: Régularité des applications faiblement harmoniques entre une surface et une variété riemannienne. C.R. Acad. Sci. Paris Sér. I Math. 312, 591-596 (1991)

7. Hong, M.-C., Wang, C.Y.: Regularity and relaxed problems of minimizing biharmonic maps into spheres. Preprint

8. Lin, F.H.: Une remarque sur l'application $x /|x|$. C.R. Acad. Sci. Paris Sér. I Math. 305, 529-531 (1987)

9. Morrey C.B. Jr.: The problem of Plateau on a Riemannian manifold. Ann. Math. 49, 807-951 (1948)

10. Price, P.: A monotonicity formula for Yang-Mills fields. Manuscripta Math. 43, 131$166(1983)$

11. Rivière, T.: Everywhere discontinuous harmonic maps into spheres. Acta Math. 175, 197-226 (1995)

12. Schoen, R.: Analytic aspects of the harmonic map problem. Seminar on Nonlinear Partial Differential Equations (S.S. Chern editor), MSRI Publications, vol. 2, Springer, New York, 1984

13. Schoen, R., Uhlenbeck, K.: A regularity theory for harmonic maps. J. Differential Geometry 17, 307-335 (1982)

14. Wang, C.Y.: Biharmonic maps from $\mathbb{R}^{4}$ into a Riemannian manifold. Math. Z. (to appear)

15. Wang, C.Y.: Stationary biharmonic maps from $\mathbb{R}^{m}$ into a Riemannian manifold. Comm. Pure Appl. Math. 57, 419-444 (2004) 